\title{
Glossary
}

\section{Glossaries in public health: older people}

\author{
A Bowling, S Ebrahim
}

Humanity is growing older. The falls in mortality and fertility rates occurring in all but sub-Saharan African countries have brought the issue of population aging, and the associated changes in disease burdens and health care provision, into the limelight. Population aging represents one of the most profound social, health and economic challenges facing us. Research in this area has been under-valued and under-funded for decades but despite this, important concepts have been developed and a vocabulary has emerged that may be unfamiliar to scientists across different disciplines. Researchers in this area are drawn from a wide range of parent disciplines and bring a richness of research methods, but this may be confusing to the uninitiated. For these reasons, we think that a glossary of widely used terms may be of value to both scientists and policy makers, as well as to the new recruits and those who want to read and better understand our work.

Activities of daily living (ADL): personal care tasks such as eating/drinking, washing self, using the toilet, rising from a chair, getting in/out of bed, moving around indoors, dressing, walking outdoors.

Activity, or role, theory: a theory emphasising the importance of pertinent social roles in determining individual identity, behaviour and social functioning. It is argued that involvement in new social roles, and activities that are meaningful to people, enhances feelings of well being and self esteem in older age.

Age based health service rationing: the imposition of upper age cut offs, sometimes representing retirement age, or digit preference, on provision of health services in order to contain health care costs.

Age cohort modelling, period cohort modelling: examination of secular trends in incidence or mortality using mathematical modelling to examine contribution of age, time period and birth cohort to observed trends. Age, period and cohort are linearly dependent, so a full model is unable to identify a unique solution for all three effects simultaneously.

Age standardisation: application of weighted averaging of rates specific for age when making comparisons. Direct standardisation applies observed age specific rates in a study population to the age distribution of a standard population; indirect standardisation applies age specific population rates to the age distribution of the study population, comparing observed with expected events. Age standardisation is the most common form of standardisation used when making comparisons between places, time periods or populations where differences in age structure of the groups compared may produce marked differences in crude unstandardised rates. It is now common to standardise for single years of age when comparing rates in older age groups as even small differences within five year age bands may influence comparisons.

Aging: A series of progressive and irreversible biological changes, which result in reduced ability to process, starts in adolescence or early adulthood, the pace of deterioration of different body systems varies considerably, and is influenced by behavioural and socioeconomic forces.

Agism: prejudice based on age, associated with the stereotyping of older people as being frail, mentally incompetent and dependent; also includes the social pressures that reduce the status and roles of older people

Autonomy: the ability of a person to make decisions for themselves and implement these-often incorrectly used synonymously with independence.

Bed blocker: derogatory term applied to older patients who outstay their welcome in acute hospital beds but usually a function of inadequate services for elderly people.

Biological theories of aging: these fall into the following major categories: "damage" theories supported by observed declines in DNA repair enzymes; genetic theories supported by heritability studies, progeria syndromes, and animal studies; disposable soma theory supported by variation in species specific life expectancies that vary depending on the relative amounts of energy put into maintenance of the body (soma) and into reproduction.

Carer: a person who supports and has most contact with a dependent older person and is not paid for their work.

Centenarian: a person surviving to their 100th birthday. A considerable achievement in the past but now more commonplace.

Chronological age: the use of specific birth anniversaries to define old age; this is increasingly regarded as inadequate for this purpose (for example, this is illustrated in the inconsistency that until recently, the official retirement age in Britain was lower for women (60) than 
men (65), while the longevity of women is greater than that of men). (See Old age).

Community care: the policy of providing social support (and health care) to a majority of older people in their own homes rather than in an institution.

Competency: term used in legal situations where a person's competency to change a will, look after their affairs, or be tried for criminal actions is in doubt. Careful mental state testing is required, often repeated, to determine competency. In those people with problems various legal protections can be organised.

Compression of morbidity: the hypothesis that morbidity in old age is being compressed into a shorter time span, perhaps because of healthier lifestyles or medical advances. Alternative theories of expansion of morbidity and of dynamic equilibrium have also been proposed.

Day hospitals: aim to provide a range of medical, nursing and rehabilitation assessments and treatments to patients who attend up to five times a week. Only limited evidence exists to support their effectiveness.

Dementia syndromes: progressive, global and irreversible cognitive impairments caused most commonly by Alzheimer's disease (linked with apoE4 polymorphism), multiple cerebral infarcts, or a combination of these.

Demographic transition: change in a society from a situation of high mortality and fertility to one of low mortality and fertility, resulting in a shift from a low to a high proportion of older people, and an increase in total population size.

Demographic timebomb: the demands made by a growing population of elderly people will outstrip health and social services provision; after three decades of concern no evidence supports this concept.

Dependency: the extent to which a person needs help from others to maintain a normal life; distinct from autonomy.

Dependency ratio: ratio of (potentially) economically active population to the retired population and children under 16 years of age. Aged dependency ratio is the ratio of economically active to retired population.

Diogenes syndrome: named after the philosopher who lived in a jar-a person who lives, usually alone, in a state of squalor that frequently gives rise to concerns and demands for resettlement elsewhere. Such people are usually eccentric rather than mentally ill.

Disability: inability to perform tasks in a normal manner. (See Activities of daily living and Instrumental activities of daily living).

Disability free life expectancy: the number of years of projected life expectancy that will be spent free of disability. Usually defined from age 65 or as a percentage of total life expectancy. (See Health Expectancy).

Disengagement theory: an outdated theory, which emphasises the discontinuity between older age and earlier stages of the life cycle. It postulates that withdrawal from social interactions and activities is an inevitable accompaniment of older age as people become more self preoccupied. The alleged functions of disengagement from society are to protect the individual against the trauma of dying and to minimise disruption to society when death takes place. Therefore it is necessary for the continuous functioning of society to expel older people (who have an increased risk of dying) from work and other social roles. There is little evidence to support this contentious theory, and it is generally criticised because it legitimises the social redundancy of older people.

Dynamic equilibrium: in relation to gerontology, this can be defined specifically as the ages at which disease and disability strike and move in tandem with the increase in life expectancy. This results in no increase (or decrease) in the proportion of life expectancy spent in ill health or disability.

Elder abuse: the severe end of a spectrum of inadequate care that includes both physical, mental and verbal abuse. Frequently associated with increased dependency and personality problems in the carer.

Elderly, the: frequently used but should be avoided as it tends to stereotype and dehumanise-“elderly people" or "older people" are preferable.

Epidemiologic transition: in parallel with demographic transition, patterns of mortality also shift from a high burden from infectious diseases to an increase in chronic degenerative diseases (cardiovascular diseases and cancers) and morbidity patterns also show a similar increase in non-fatal but disabling conditions (for example, osteoarthritis).

Ethnic elders: with increased migration it is much more common for people to become old in a country not their own: thought to bring increased stress because of racial prejudice, poorer access to services than the majority population, and aging itself.

"Fair innings" argument: it is argued, on the basis of utilitarianism - that is, the greatest good of the greatest number - that older people have had a "fair innings" and should give up their place in the health service queue; instead scarce medical resources should be diverted towards the young, in order to give them a chance of reaching older age.

Fitness gap: the difference between what a person is able to do (for example, climb stairs) and needs to do to be able to maintain independence, sometimes referred to as the disability gap or ecological gap.

Fourth age: the period of increasing frailty before death.

Geriatric giants: the common problems that often affect older people-mobility problems, confusion, falls, incontinence of urine, medication problems, hearing and vision impairments (sometimes known as the five "Is" immobility, incontinence, instability, inability, insanity iatrogenesis).

Gompertz equation: one of a range of mathematical descriptions of the shape of the survival curve in old age. Death rates only reach an almost exponential function with age beyond 95 years.

Grey panthers: old age activists in USA who have successfully campaigned for better services for older people. 
Handicap: is the social disadvantage or loss of role associated with disease. It is possible for impairment to result in handicap independent of disability (for example, port wine birthmark may cause social handicap without any disability). (See Impairment, Disability and Handicap).

Health expectancy: a generic term for the wide range of indicators of life expectancy adjusted for health status (for example, dementia free life expectancy, disability free life expectancy, healthy active life expectancy).

Health related quality of life: like quality of life, this is an amorphous concept and a wide range of pertinent domains have been identified in the literature, including the perceived impact of health on optimum levels of physical, psychological and social well being and functioning, level of independence and control over life, and satisfaction with these levels.

Health status: perceived physical, psychological and social health.

Healthy active life expectancy (HALE): the average number of years that a fit person of a given age can be expected to have before experiencing disability.

Health services for elderly people: four main models of provision exist: needs related in which elderly people are managed by geriatricians and their teams according to their need for a team approach; age related in which all people over a certain age $(75+$ or $85+)$ are managed by geriatricians; integrated services in which consultants with a special interest in geriatric medicine take a full role in acute internal medicine duties and also provide needs related care to those older people who require a team approach; and integration between geriatric and old age psychiatry services.

Impairment: is the anatomical or physiological damage caused by disease (for example, reduction in cardiac output caused by ischaemic heart disease, restriction in joint movement caused by osteoarthritis). (See Impairment, Disability and Handicap).

Impairment, Disability and Handicap: The World Health Organisation (International Classification of Impairments, Disabilities and Handicaps. Geneva: WHO, 1980) made these important distinctions between the ways in which chronic diseases have an impact on the individual. This classification has since been revised to Impairments, Activities and Participation as the terms disability and handicap may be viewed as stigmatising by some people. (World Health Organisation ICIDH-2. Geneva: WHO, 1998).

Instrumental activities of daily living (IADL): household, rather than personal, management activities: preparing meals, bed making, laundry/ironing, managing money, using the telephone, shopping and heavy housework.

Life expectancy: is the average (median) number of years that a person can expect to live but is usually based on contemporary death rates that may be higher than those experienced by future cohorts, so tends to underestimate life expectancy. May be calculated from birth (in which case it is dominated by infant mortality in poorer countries), or from other ages.

Life span: is the maximum number of years that a person has been known to live, currently around 115 years. Frequently confused with life expectancy.

Life table: a summary measure of the mortality and survival of a population. Current life tables summarise mortality experience by age of a population at a point in time. Cohort life tables summarise the actual mortality experience of a cohort born at about the same time and followed until all are dead. The $\mathrm{e}^{0}{ }_{x}$ function derived from a life table is the average number of years of life remaining to people who survive to age $x$.

Limiting longstanding illness: a simple question frequently used in government surveys and censuses in an effort to estimate the prevalence of disability. Addition of the words "including problems due to old age" increases the prevalence markedly.

Living wills: or advance directives are legal statements (or sometimes, contracts with medical services) drawn up before the onset of chronic disabling disease that may impair judgement and autonomy (for example, dementia) that define a person's wishes for health and social care. Their standing in law is not clear and they may be not be implementable.

Modernisation theory: a controversial theory holding that, at the same time as demographic transition, modernisation of society occurs that undermines the status of older people (for example, through the introduction of new technology, which undermines their skills; through the emphasis on education, which undermines their traditional role as teachers of knowledge and skills; through the impact of urbanisation disrupting family life).

Multiple pathology: is the coincident occurrence of more than one pathological process in an older person. This is sometimes used as a justification for exclusion of older people from treatment trials and in epidemiological studies.

Old age: this is usually defined in relation to a specific birth anniversary, often coinciding with an official age of retirement. (See chronological age.) But like current retirement ages, this definitional age cut off is arbitrary and increasingly blurred with the increase in early retirement and unemployment among older workers. Terms such as the "old old" and the "oldest old" have been used to refer to those aged $75+$ and $85+$ respectively.

Old age psychiatry: a new service that has developed dramatically in response to the needs of older people and is concerned with both functional mental illnesses and dementias.

Pseudodementia: cognitive impairment associated with depression that usually improves with treatment of the underlying depression.

Quality of life: although this is a multi-level and amorphous concept, it can be broadly defined as encompassing the person's perceptions of, and satisfaction with, physical health, psychological well being (for example, anxiety and depression), independence, social relationships, social and material circumstances and 
the external environment (the natural and built environments). It is a concept that is dependent on the perceptions of people. Perceived quality of life is arguably mediated by several interrelated variables, including self related constructs (for example, self efficacy, self esteem, perceived control over life) and subjective evaluations of quality of life are theoretically influenced by cognitive mechanisms (for example, expectations of life, social values and beliefs, aspirations and standards for social comparisons of one circumstances in life).

Rectangularisation of survival: the change in the shape of the population survival curve in the 19 th century that featured high infant mortality to the more rectangular shape associated with very high survival prospects in early life.

Rehabilitation: formally defined as the action of re-establishing a person in a former standing with respect to rank and legal rights and, in the context of medicine, is concerned with reablement of a person through provision of a stimulating environment, and encouraging greater activity, participation and autonomy; and re-settlement either in the person's own home or in alternative, more sheltered accommodation. Often necessary for older people after a short acute illness.

Respite care: the temporary admission of a dependent older person to an institution to provide both a period of rest (or respite) for the carer and an opportunity for palliative or rehabilitative care for the person.

Retirement age: inability or choice not to remain in paid employment associated with reaching a certain age (varies between 50 and 70 years). Increasingly, retirement ages are more flexible.

Section 47: part of the UK National Assistance Act (1948) that permits the removal of a person from their home because they are a danger to themselves or others; use of this section is not subject to the same controls as Mental Health Act section orders and is open to abuse.

Social construction of old age: the definition of old age, and the experiences of older people, as a product of the way society is organised and the structure of inequality within it, rather than a natural concomitant of the aging process (for example, as in the traditional definition of old age that coincides with official retirement age).

Social capital: affordable and accessible leisure and local transport facilities, food, the convenience, safety and people friendly nature of the local environment, social and community integration.

Social handicap: barriers to participation and independent functioning imposed by society, as in the case of the built environment and public transport not taking degenerative changes of older age into account in their design.

Social network: the set of links between identified groups of people.

Social support: the interactive process through which emotional and instrumental aid, information and guidance is received from a person's social network.

Structured dependency: dependency in old age is structured by the dominant economic and political forces (for example, the timing, experience and outcome of retirement is related to the supply and demand for labour; welfare services are said to stigmatise older people though the imposition of age related policies).

Successful, or positive, aging: growth and development in older age, and the successful adaptation to losses and challenges associated with increasing age (for example, using psychological strategies, physical and social resources).

Third age: a continuation of life in which retirement enables citizens to live at leisure and realise their purposes. This option is not available for people on low incomes or in poor health.

Utilitarianism: production of the greatest possible total happiness, good, or "utility", of the greatest number of people. Utilitarian philosophy is often invoked to defend age based health care rationing policies. This disregards the principle of equity in access to health care.

University College London is a member of the MRC Health Services Research Collaboration, of which Bristol is a lead centre.

\section{Further reading}

Ebrahim S, Kalache A, eds. Epidemiology in old age. London: BMJ Publishing Group, 1996.

Baltes PB, Baltes MM. Successful aging:perspectives from the behavioural sciences. New York: Cambridge University Press, 1990.

Bowling A. The concepts of successful and positive ageing. Fam Pract 1993; 10:449-53.

Fennell G, Phillipson C, Evers H. The sociology of old age. BuckFennell G, Phillipson C, Evers H. The

Last JM. A dictionary of epidemiology. 2nd ed. Oxford: Oxford University Press, 1988.

Rothman KJ, Greenland S. Modern epidemiology. 2nd ed. Philadelphia: Lippincott Williams and Wilkins, 1998.

Tallis RC, Fillit HM, Brocklehurst JC. Brocklehurst's textbook of geriatric medicine and gerontology. 5th ed. Edinburgh: Churchill Livingstone, 1998. 